\title{
The diagnostic value of the serum irisin level in patients with acute pericarditis and acute myopericarditis
}

\author{
Gunaydin $\mathrm{M}^{1}$, Ozer $\mathrm{V}^{2}$, Kalkan $\mathrm{A}^{3}$, Ozer $\mathrm{S}^{4}$, Sahin $\mathrm{A}^{5}$, Sahin $\mathrm{M}^{6}$, Karahan $\mathrm{SC}^{7}$, Dogramaci $\mathrm{S}^{7}$, \\ Tatli $\mathrm{O}^{5}$, Gunduz $\mathrm{A}^{5}$ \\ Giresun University, Faculty of Medicine, Department of Emergency Medicine, Giresun, Turkey. \\ mgunaydin@hotmail.com
}

\begin{abstract}
OBJECTIVE: This preliminary study aims to examine a change in the blood levels of irisin in patients with acute pericarditis (AP) and acute myopericarditis (AMP) and examine the diagnostic value of the serum irisin level in AP and AMP.

METHODS: 10 patients, who applied to the emergency service and cardiology clinic with chest pain and who were diagnosed with AP and 5 patients, who were diagnosed with AMP as a result of routine examinations, were included in the study. The basal laboratory parameters, echocardiography findings and serum irisin levels of the patients and during check one month later were examined.

RESULTS: While the basal irisin levels were found to be significantly low in the AMP group and high during the check $(6.6 \pm 1.58,8.19 \pm 1.43$, respectively), no statistically significant difference was determined $(p=0.23)$. It was observed that the basal and control irisin levels did not vary significantly in the AP group $(8.03 \pm 1.6,8.19$ \pm 1.43 , respectively) $(p=0.84)$.

CONCLUSION: In this preliminary study, the basal irisin levels were found to be significantly low in the AMP group, while there was no statistically significant difference between the basal irisin levels and control irisin levels in the AP and AMP groups (Tab. 5, Ref. 17).

KEY WORDS: irisin, pericarditis, myopericarditis.
\end{abstract}

\section{Introduction}

Pericarditis is the inflammation of the pericardial sac, and it is more prevalent among males (20-50 years of age). While myocarditis is the name for cases in which the pericardium is dominantly affected, but some involvement is observed in the myocardium, cases in which myocardial involvement is in the foreground, but the pericardium is also affected a bit, are called myopericarditis (1, 2 ). The most prevalent cause of pericarditis in developed countries is idiopathic viral and bacterial infections at a rate of $65-80 \%$, and viral causes are more prevalent. The history of cardiac surgery, the history of percutaneous coronary intervention, systemic inflammatory conditions, and renal insufficiency are responsible for non-ineffective cases $(2,3)$. Parameters such as electrocar-

${ }^{1}$ Giresun University, Faculty of Medicine, Department of Emergency Medicine, Giresun, Turkey, ${ }^{2}$ Rize State Hospital, Department of Emergency Medicine, Rize, Turkey, ${ }^{3}$ Haseki Training and Research Hospital, Department of Emergency Medicine, İstanbul, Turkey, ${ }^{4}$ Recep Tayyip Erdogan University, Faculty of Medicine, Department of Cardiology, Rize, Turkey, ${ }^{5}$ Karadeniz Technical University, Faculty of Medicine, Department of Emergency Medicine, Trabzon, Turkey, ${ }^{6}$ Karadeniz Technical University, Faculty of Medicine, Department of Cardiology, Trabzon, Turkey, and ${ }^{7}$ Karadeniz Technical University, Faculty of Medicine, Department of Biochemistry, Trabzon, Turkey

Address for correspondence: M. Gunaydin, Giresun Universitesi Tip Fakultesi, Gazipasa Yerleskesi, Debboy Mevkii, 28100, Giresun, Turkey. Phone: +90.454.3101600, Fax: +90.454.3101699 diography (ECG), echocardiography (ECHO), cardiac magnetic resonance imaging (MRI), tomography $(\mathrm{CT}), \mathrm{C}$-reactive protein (CRP), erythrocyte sedimentation rate (ESR), and antinuclear antibody (ECG) are used in the diagnosis of acute pericarditis $(2,3)$. Necessary examinations should be made to exclude acute coronary syndrome in cases, which are believed to have myocardial involvement and in which the cardiac enzyme increases (2).

Irisin acts as adenosine triphosphate (ATP) sensor according to the energy need of the local tissue. In case the tissue does not need energy, the existing energy is turned into heat with the amount of irisin at physiological levels. If the tissue needs ATP, then, irisin production in the tissue decreases. Furthermore, it is believed that the low level of irisin protects cells against necrosis to some extent by inhibiting consumption by means of turning ATP into heat (4, 5). In animal experiments, in which acute myocardial infarction was induced, it was understood that the blood irisin level decreased in order to prevent the energy need in the body, and this created a cardioprotective effect $(6,7)$. In acute pericarditis (AP) and acute myopericarditis (AMP), it can be assumed that the blood irisin level may decrease and cause a cardioprotective effect due to the need for energy in the body. Knowing the level in these patients may give us information about the relationship between the energy metabolism of the patient and irisin and cardiac-based diseases.

In this study, it was planned to determine the change of the blood levels of irisin, which is a new peptide, in AP and AMP patients at the beginning and after the treatment (1 month later), and 
655-659

investigate the usability of irisin in the diagnosis and treatment monitoring of AP and AMP.

\section{Materials and methods}

\section{Study population}

After receiving the ethics committee approval, it was planned to include patients, who applied to the emergency service and cardiology clinic of the University Faculty of Medicine with chest pain complaints, who were diagnosed with AP or AMP with the routine laboratory, electrocardiography and echocardiography findings, who gave their written consent for the participation in the study, and who were at the age of 18 and above. The presence of moderate and severe heart valve disease, heart valve prosthesis, congenital heart disease, hypertrophic cardiomyopathy, and persistent pacemaker, the fact that the ejection fraction was lower than 50 $\%$, the presence of uncontrolled or untreated thyroid dysfunction, pregnancy, renal failure, acute and chronic liver disease, and the presence of an additional focus of infection apart from pericarditis and myopericarditis, the history of malignity, and diabetes mellitus were determined to be the exclusion criteria.

In this study, the number of target cases was determined to be approximately 20, since the incidence of pericarditis was low. 32 patients, who were diagnosed with AP and AMP, were assessed. 17 patients were excluded from the study due to various reasons ( 2 patients with moderate and severe valve disease, 3 patients with diabetes mellitus, 4 patients with serum creatinine $>1.2 \mathrm{mg} / \mathrm{dL}$, and 8 patients that did not come to the follow-up). The study was conducted with 15 patients in total, after removing the excluded patient group.

The demographic features of all patients at the moment of application were recorded. The height and weight of the patients were measured using the NAN (Turkey) height and weight scale. The body mass indices (BMI) of the patients were calculated and recorded.

About $2 \mathrm{ml}$ of the blood taken from the patients into a biochemistry tube at the moment of application and for routine laboratory tests was separated in order to examine the irisin levels from the serum samples. Routine ECG was applied to these patients for the AP and AMP diagnosis, and the specified measurements were made by performing ECHO. 5 patients, who were thought to have myocardial involvement and who had elevated cardiac enzyme, underwent coronary angiography (CAG) in order to exclude acute coronary syndrome, and these patients with normal CAG results were regarded to have AMP. It was observed that the troponin values of the remaining 10 patients were within normal limits, and these patients were regarded to have AP. Patients diagnosed with AP and AMP were assessed in two separate groups, and the statistical values were calculated separately.

\section{Echocardiographic evaluation}

The echocardiographic measurements of the patients were performed transthoracically by a Vivid 7 (GE Vingmed Ultrasound, Horten, Norway) ECHO device. The left atrium diameter (LA), left ventricular systolic end and diastolic end diameters (LVSD and
LVDD), septum and posterior wall thicknesses (IVS and PW), as well as epicardial fat thickness, were measured from the parasternal long axis image. The ejection fraction (LVEF) was calculated from the apical four-cavity image by the modified Simpson's method. The right ventricular (RV) and right atrial (RA) diameters were measured. The pulmonary artery pressure (sPAB) was measured over the tricuspid valve jet with continuous Doppler flow (CW).

\section{Biochemical parameters}

About $2 \mathrm{ml}$ of the blood taken from the patients into a biochemistry tube at the moment of application for routine laboratory tests was separated in order to examine the serum irisin levels from the serum samples. About $2 \mathrm{ml}$ of the blood taken from the patients in the study group into a biochemistry tube during the check held one month after the start of the treatment for routine laboratory tests was separated in order to examine the serum irisin levels from the serum samples.

The glucose, creatinine, CRP, haemoglobin, white blood cell and platelet count, troponin, $\mathrm{CK}-\mathrm{MB}$, thyroid stimulating hormone (TSH), T4, low-density lipoprotein (LDL), high-density lipoprotein (HDL), total cholesterol and triglyceride levels in the serum samples taken from the patients were recorded.

\section{Serological analysis}

Determination of human serum irisin levels

The irisin levels in human serums were determined in accordance with the manufacturer's recommendations using an enzymelinked immunosorbent assay (ELISA) kit (Elabscience, Cat No: RAG018R, Lot: X17-001, Czech Republic).

The transfer of the samples into the ELISA plate and their preparation for the measurement

Serums kept at $-80{ }^{\circ} \mathrm{C}$ were brought to room temperature. The irisin standards were prepared in accordance with the kit procedures and loaded into the wells to be $50 \mu \mathrm{L}$. After the dilution of the samples at the rate of $1 / 4,50 \mu \mathrm{L}$ was taken and transferred to the wells separated for the samples in the ELISA plate. $50 \mu \mathrm{L}$ of the Detection Antibody was added to each well. The plate was covered with tinfoil and left for incubation in the shaker for 60 minutes at $37{ }^{\circ} \mathrm{C}$. The plate was washed 3 times with the washing buffer and plate washer after the incubation. The remaining residue in the wells was removed with the washing buffer, and $100 \mu \mathrm{L}$ HRP Conjugated anti-rabbit IgG solution was added to each well. The plate was recovered with tinfoil and left for incubation for 60 minutes in the shaker at $37^{\circ} \mathrm{C}$. After the incubation, the plate was washed 5 times with the washing buffer and plate washer. The remaining residue in the wells was removed with the washing buffer

\section{Staining of the samples and measurement}

$100 \mu \mathrm{L}$ of TMB Substrate was added to each well for staining. It was left for incubation for 15-20 minutes in the dark at room temperature. $100 \mu \mathrm{L}$ of staining stop solution was added to each well as soon as the colour of the standards turned into blue, and it was observed that the colour of the samples turned into yellow. 
The absorbents of the samples were measured at a wavelength of $450 \mathrm{~nm}$ in a VERSA (Designed by molecular Devices in California, USA) brand micro-plate reader. The results are given in $\mu \mathrm{g} / \mathrm{mL}$.

\section{Statistical analysis}

SPSS 23.0 statistical package program was used in the stage of data analysis. The descriptive statistics of the evaluation results were presented as number and percentage for categorical variables, and mean, standard deviation, minimum, maximum values for numerical variables. The normal distribution of numerical variables was evaluated by the Shapiro-Wilk test. The paired t-test was used since the condition of normal distribution was not provided in the comparison of the numerical variables between the two dependent groups. Regarding correlation analyses, Pearson's correlation test was used in the data exhibiting normal distribution, and Spearman's correlation test was used in the data not exhibiting normal distribution. The statistical significance level was accepted as $\mathrm{p}<0.05$.

\section{Results}

The demographic characteristics of the patients diagnosed with AP and AMP, who were included in the study are presented in the Table 1. No statistically significant difference was determined, when the basic demographic data of the patients with AP and AMP were compared.

The basal biochemical data of the patients with AP and AMP are presented in the Table 2. When the basal biochemical data of the patients with AP and AMP were compared, no statistically significant difference was detected in the data except for the CRP and troponin value. While the mean CRP value of the patients with AP was $1.17 \pm 1.95$, the mean CRP value of the patients with AMP was found to be $10.6 \pm 5.9$. While the mean troponin value of the patients with AP was $10.19 \pm 13.04$, the mean troponin value of the patients with AMP was found to be $487.18 \pm 248.4$. It was found out that the CRP and troponin values between the two groups were statistically significant ( $\mathrm{p}=0.001$ and $\mathrm{p} \leq 0.001$, respectively).

The basal ECHO data of AP and AMP patients are presented in Table 3. When the ECHO data of the patients with AP and AMP

Tab. 1. Demographic characteristics of AP and AMP groups.

\begin{tabular}{lccc}
\hline & $\begin{array}{c}\text { AP group } \\
(\mathrm{n}=10)\end{array}$ & $\begin{array}{c}\text { AMP group } \\
(\mathrm{n}=5)\end{array}$ & $\mathrm{p}$ \\
\hline Age (years) & $24.5 \pm 9.26$ & $26.8 \pm 11.6$ & 0.768 \\
\hline Gender & $2(20 \%)$ & $1(20 \%)$ & 1.0 \\
$\quad \begin{array}{l}\text { Female }(\%) \\
\text { Male }(\%)\end{array}$ & $8(80 \%)$ & $4(80 \%)$ & \\
\hline BMI $\left(\mathrm{kg} / \mathrm{m}^{2}\right)$ & $24.3 \pm 4.87$ & $24.6 \pm 3.78$ & 0.906 \\
\hline Hyperlipidemia $(\%)$ & $0(0 \%)$ & $1(20 \%)$ & 0.333 \\
\hline Coronary Artery Disease $(\%)$ & $0(0 \%)$ & $0(0 \%)$ & - \\
\hline Hypertension $(\%)$ & $0(0 \%)$ & $1(20 \%)$ & 0.333 \\
\hline Diabetes Mellitus $(\%)$ & $0(0 \%)$ & $0(0 \%)$ & - \\
\hline Smoking $(\%)$ & $4(40 \%)$ & $2(40 \%)$ & 1.0 \\
\hline Stroke/GIA history $(\%)$ & $0(0 \%)$ & $0(0 \%)$ & - \\
\hline Heart failure $(\%)$ & $0(0 \%)$ & $0(0 \%)$ & - \\
\hline
\end{tabular}

Tab. 2. Basal biochemical data of patients of AP and AMP groups.

\begin{tabular}{lccc}
\hline & $\begin{array}{c}\text { AP group } \\
(\mathrm{n}=10)\end{array}$ & $\begin{array}{c}\text { AMP group } \\
(\mathrm{n}=5)\end{array}$ & $\mathrm{p}$ \\
\hline Glucose $\mathrm{mg} / \mathrm{dL}$ & $101.8 \pm 23.5$ & $100.4 \pm 7.4$ & 0.9 \\
\hline Creatine $\mathrm{mg} / \mathrm{dL}$ & $0.78 \pm 0.18$ & $0.74 \pm 0.11$ & 0.663 \\
\hline Total cholesterol mg/dL & $165.8 \pm 36.6$ & $168.2 \pm 70.5$ & 0.699 \\
\hline Triglyceride $\mathrm{mg} / \mathrm{dL}$ & $106.2 \pm 76.2$ & $94 \pm 26.4$ & 0.898 \\
\hline LDL $\mathrm{mg} / \mathrm{dL}$ & $105.1 \pm 24.9$ & $113.2 \pm 52.8$ & 0.724 \\
\hline HDL $\mathrm{mg} / \mathrm{dL}$ & $42 \pm 6.2$ & $36.2 \pm 18.8$ & 0.083 \\
\hline Haemoglobin & $14.6 \pm 1.5$ & $14.2 \pm 1.7$ & 0.657 \\
\hline White blood cell & $8820 \pm 4358$ & $11500 \pm 3762$ & 0.263 \\
\hline Platelet & $229900 \pm 58572$ & $312600 \pm 222179$ & 0.456 \\
\hline TSH $\mu \mathrm{IU} / \mathrm{mL}$ & $1.76 \pm 1.18$ & $2.12 \pm 1.86$ & 0.659 \\
\hline sT4 ng/dL & $1.16 \pm 0.27$ & $1.34 \pm 0.26$ & 0.239 \\
\hline C-reactive protein $\mathrm{mg} / \mathrm{dL}$ & $1.17 \pm 1.95$ & $10.6 \pm 5.9$ & 0.001 \\
\hline Troponin & $10.19 \pm 13.04$ & $487.18 \pm 248.4$ & $<0.001$ \\
\hline
\end{tabular}

Tab. 3. Basal ECHO parameters of AP and AMP groups.

\begin{tabular}{lccc}
\hline & $\begin{array}{c}\text { AP group } \\
(\mathrm{n}=10)\end{array}$ & $\begin{array}{c}\text { AMP group } \\
(\mathrm{n}=5)\end{array}$ & $\mathrm{p}$ \\
\hline Ejection Fraction $(\%)$ & $64.7 \pm 1.8$ & $59.8 \pm 5.1$ & 0.099 \\
\hline LVESD $(\mathrm{mm})$ & $30.9 \pm 3.4$ & $31.8 \pm 1.5$ & 0.585 \\
\hline LVEDD $(\mathrm{mm})$ & $45.6 \pm 3.6$ & $44.8 \pm 0.8$ & 0.859 \\
\hline LA $(\mathrm{mm})$ & $33.6 \pm 3.5$ & $34 \pm 2.3$ & 0.821 \\
\hline IVS & $9.5 \pm 1.43$ & $9.8 \pm 1.48$ & 0.803 \\
\hline PW $(\mathrm{mm})$ & $8.9 \pm 1.2$ & $9.2 \pm 1.6$ & 0.692 \\
\hline RV $(\mathrm{mm})$ & $32.7 \pm 3.1$ & $31.6 \pm 4.3$ & 0.581 \\
\hline RA $(\mathrm{mm})$ & $34.9 \pm 2.5$ & $35.4 \pm 2.9$ & 0.731 \\
\hline Spab & $21.2 \pm 2.6$ & $20.4 \pm 0.9$ & 0.953 \\
\hline
\end{tabular}

Tab. 4. Serum Irisin levels $\mu \mathrm{g} / \mathrm{ml}$ of AP and AMP groups

\begin{tabular}{lccc}
\hline & Basal irisin level & Control irisin level & $\mathrm{p}$ \\
\hline AP group $(\mathrm{n}=10)$ & $8.03 \pm 1.6$ & $8.19 \pm 1.43$ & 0.84 \\
\hline AMP group $(\mathrm{n}=5)$ & $6.6 \pm 1.58$ & $8.18 \pm 1.69$ & 0.23 \\
\hline $\mathrm{p}$ & 0.127 & 0.986 & \\
\hline
\end{tabular}

Tab. 5. Correlations between the basal biochemical data and the basal irisin levels of the patients in the AP and AMP groups.

\begin{tabular}{lccccc}
\hline & \multicolumn{2}{c}{$\begin{array}{c}\text { AP Group } \\
(\mathrm{n}=10)\end{array}$} & & \multicolumn{2}{c}{$\begin{array}{c}\text { AMP Group } \\
(\mathrm{n}=5)\end{array}$} \\
\cline { 2 - 3 } \cline { 6 - 7 } & $\mathrm{r}$ & $\mathrm{p}$ & & $\mathrm{r}$ & $\mathrm{p}$ \\
\hline Glucose $\mathrm{mg} / \mathrm{dL}$ & 0.879 & 0.001 & & 0.203 & 0.743 \\
\hline Creatine $\mathrm{mg} / \mathrm{dL}$ & 0.05 & 0.892 & & -0.315 & 0.605 \\
\hline Total cholesterol $\mathrm{mg} / \mathrm{dL}$ & 0.07 & 0.859 & & -0.9 & 0.037 \\
\hline Triglyceride $\mathrm{mg} / \mathrm{dL}$ & -0.083 & 0.831 & & -0.1 & 0.873 \\
\hline LDL $\mathrm{mg} / \mathrm{dL}$ & 0.097 & 0.819 & & -0.9 & 0.037 \\
\hline HDL $\mathrm{mg} / \mathrm{dL}$ & 0.377 & 0.318 & & -0.3 & 0.624 \\
\hline Haemoglobin & -0.274 & 0.444 & & 0.934 & 0.02 \\
\hline White blood cell & 0.499 & 0.142 & -0.552 & 0.335 \\
\hline Platelet & -0.131 & 0.719 & & -0.836 & 0.078 \\
\hline TSH $\mu \mathrm{IU} / \mathrm{mL}$ & -0.343 & 0.366 & & 0.386 & 0.521 \\
\hline sT4 $\mathrm{ng} / \mathrm{dL}$ & -0.221 & 0.568 & & 0.32 & 0.6 \\
\hline C-reactive protein $\mathrm{mg} / \mathrm{dL}$ & 0.609 & 0.061 & -0.161 & 0.795 \\
\hline Troponin & 0.238 & 0.508 & & -0.373 & 0.537 \\
\hline Age $($ years) & -0.14 & 0.7 & -0.359 & 0.553 \\
\hline BMI $\left(\mathrm{kg} / \mathrm{m}^{2}\right)$ & -0.281 & 0.432 & -0.471 & 0.471 \\
\hline
\end{tabular}


were compared, no statistically significant difference was determined between the two groups.

The serum irisin levels of the patients in the AP and AMP groups measured during the diagnosis and the serum irisin levels measured during the period, when they recovered 1 month later are presented in Table 4. It was observed that the initial irisin levels of the patients diagnosed with AMP were significantly low and increased during the check held 1 month later $(6.6 \pm 1.58,8.19$ \pm 1.43 , respectively), while the initial and control irisin levels of the patients diagnosed with AP did not change significantly (8.03 $\pm 1.6,8.19 \pm 1.43$, respectively). Nevertheless, no statistically significant difference was determined between the irisin levels of the patients at the time, when they were diagnosed with AP and AMP and the irisin levels measured after they recovery $(\mathrm{p}=0.84$, $\mathrm{p}=0.23$, respectively). When the basal and control irisin levels of AP and AMP patients were compared, no statistically significant difference was determined ( $p=0.127, p=0.196$, respectively).

The correlation between the basal biochemical data and BMI and basal irisin levels of the patients in the AP and AMP groups are presented in the Table 5. Accordingly, a statistically significant correlation was determined between the basal glucose level and the initial irisin level in the AP group ( $\mathrm{r}=0.879, \mathrm{p}=0.001$, respectively), but no significant correlation was determined with other biochemical data. As for the AMP group, a significant correlation was determined between the basal total cholesterol, LDL and haemoglobin levels and basal irisin levels $(r=-0.9, p=0.037$; $\mathrm{r}=-0.9, \mathrm{p}=0.037 ; \mathrm{r}=0.934, \mathrm{p}=0.02$, respectively). No statistically significant correlation was determined between the initial irisin levels and BMI, Troponin, CRP and white blood cell levels in both groups.

\section{Discussion}

The fact that irisin is secreted in the body by the heart and skeletal muscle, adipose tissue, liver, kidney, peripheral nerve sheath, dermis and hypodermis in the skin has been revealed in previous studies (5). Heart muscle cells produce more irisin, when compared to skeletal muscles (8). Studies that investigate the relationship between irisin levels and myocardial infarction have been published in recent years. With the studies conducted, it was determined that low irisin levels had a protective role against the energy loss of myocardial cells $(5,7)$. The present study is the first study that has been conducted in patients with AP and AMP, which are rare infectious diseases of the heart, by investigating plasma irisin levels.

The serum irisin levels of the patients in the AP and AMP groups measured at the beginning and during the check performed 1 month later were compared in this study, and no statistically significant difference was determined in both groups. However, it was determined that the initial irisin levels of AMP patients $(6.6 \pm 1.58)$ were significantly lower, when compared to AP patients $(8.03 \pm 1.6)$ and increased during the check $(8.19 \pm 1.43)$. It was observed that irisin levels did not change in AP patients with isolated pericardial involvement. It is believed that the fact that no statistical significance occurred might result from a very low number of cases. In the study conducted by Kuloğlu et al on rats, it was observed that serum irisin levels decreased after coronary ischemia, and then returned to the old levels (5). Similarly, Aydin et al and Anastasilakis et al determined that the irisin levels were low in the studies they conducted on patients diagnosed with MI $(7,9)$. Efe et al showed that low serum irisin levels could be used in estimating the severity of coronary disease in patients with stable angina (10). These studies conducted showed that a decrease in irisin levels when the energy balance in the myocardial tissue deteriorates had a heart-protective effect. In a way that supports these studies, it is believed that the basal irisin levels are determined to be low since there is myocardial involvement in AMP and irisin is a protein secreted from the myocardium, and irisin levels did not change since there is no myocardial involvement in AP. Consequently, it may be concluded that irisin shows its cardioprotective effect in diseases that involve only the myocardium of the heart.

In this study, the correlation between the basal irisin levels and biochemical parameters and BMI was also investigated. Despite having found the troponin levels in the AMP group significantly higher, when compared to the AP group, a significant correlation between irisin levels and troponin was not determined in both groups. On the contrary, Kuloğlu et al found a significant negative correlation between low serum irisin levels and troponin in the animal MI model (5). It will be correct to expect a negative correlation between troponin and irisin in AMP patients with myocardial involvement in which troponin levels increase due to this. The result of the present study might not be reliable since the number of AMP patients in this study is very low. A significant correlation was not determined between CRP and white blood cells and irisin in both groups among inflammation parameters. While Baran et al found a positive correlation between CRP and irisin levels in patients with psoriasis, again Baran et al did not determine a significant correlation between white blood cell and irisin levels in patients with psoriasis, and Icli et al did not find a significant correlation between white blood cell and irisin levels in Bechet's disease $(11,12)$. It can be thought that the increase in CRP and white blood cells does not directly change the levels of irisin and leads to a change in irisin levels according to the area of infection.

Although certain researchers determined a positive correlation between irisin levels and BMI $(4,13)$, Moreno-Navarette et al determined a negative correlation (14). In the present study, no significant correlation was determined between the basal irisin levels and BMI in both AP and AMP groups. Sanchiz-Gomar et al, Baran et al and Icli et al did not determine any significant correlation between BMI and irisin levels in a way that supports the results of the present study $(11,12,15)$. While a significant negative correlation was determined between LDL, total cholesterol and irisin in this study, a positive correlation was determined in certain studies (16), while some did not determine a significant relationship (12).

Viral factors are mainly encountered in the aetiology of AP and AMP. The number of studies that investigate the relationship between irisin levels and infectious diseases is quite low in literature. 
In the study conducted by Buyuktuna et al, serum irisin levels were found to be high in patients with Crimean-Congo haemorrhagic fever (CCHF), and increased endothelial damage and increased haemorrhagic status were shown as the reason for this (17). In this study, serum irisin levels did not change significantly in both of the AP and AMP groups, but it was observed that the basal irisin levels were low in the AMP group, when compared to the control. Isolated heart involvement is observed in AP and AMP and does not lead to systemic findings such as CCHF. With these findings, it is understood that different values can be observed in irisin levels in infectious diseases according to the area of the factor and systemic findings it will create.

\section{Conclusion}

While there was no statistically significant result between the basal irisin levels and control irisin levels in this preliminary study in both AP and AMP groups, the basal irisin levels were determined to be significantly low in the AMP group. Based on these findings, it may be concluded that the heart may reduce serum irisin levels in diseases that involve only the myocardium, whether it is due to hypoxia or inflammation in the heart, and isolated pericardium involvement may not cause a change in serum irisin levels. However, it is necessary to conduct more comprehensive studies with a higher number of patients in order to support the results of the present study.

\section{References}

1. Shammas NW, Padaria RF, Coyne EP. Pericarditis, Myocarditis, and Other Cardiomyopathies. Prim Care 2013; 40 (1): 213-236.

2. Fardman A, Charron P, Imazio M, Adler Y. European Guidelines on Pericardial Dieases: a Focused Review of Novel Aspects. Curr Cardiol Rep 2016; 18 (5): 46.

3. Kloos JA. Characteristics, Complications, and Treatment of Acute Pericarditis. Crit Care Nurs Clin North Am 2015; 27 (4): 483-497.

4. Aydin S. Three new players in energy regulation: preptin, adropin and irisin. Peptides 2014; 56: 94-110.

5. Kuloglu T, Aydin S, Eren MN, Yilmaz M, Sahin I, Kalayci M, Sarman S, Kaya N, Yilmaz OF, Turk A, Aydin Y, Yalcin MH, Uras N, Gurel A, Ilhan S, Gul E, Aydin S. Irisin: A potentially candidate marker for myocardial infarction. Peptides 2014; 55: 85-91.
6. Boström P, Wu J, Jedrychowski MP, Korde A, Ye L, Lo JC, Rasbach KA, Boström EA, Choi JH, Long JZ, Kajimura S, Zingaretti MC, Vind BF, Tu H, Cinti S, Hojlund K, Gygi SP, Spiegelman BM. A PGC1- $\alpha$ dependent myokine that drives browing of white fat and thermogenesis. Nature 2012; 481 (7382): 463-468.

7. Aydin S, Kobat MA, Kalayci M, Eren MN, Yilmaz M, Kuloglu T, Gul E, Secen O, Alatas OD, Baydas A. Decreased saliva/serum irisin concentrations in the acute myocardial infarction promising for being a new candidate biomarker for diagnosis of this pathology. Peptides 2014; 56: 141-145.

8. Park SE, Park CY, Sweeney G. Biomarkers of insulin sensitivity and insulin resistance: Past, present and future. Crit Rev Clin Lab Sci 2015; 52: $180-190$.

9. Anastasilakis AD, Koulaxis D, Kefala N, Polyzos SA, Upadhyay J, Pagkalidou E, Economou F, Anastasilakis CD, Mantzoros CS. Circulating irisin levels are lower in patients with either stable coronary artery disease (CAD) or myocardial infarction (MI) versus healthy controls, whereas follistatin and activin A levels are higher and can discriminate MI from CAD with similar to CK-MB accuracy. Metabolism 2017; 73: 1-8.

10. Efe TH, Açar B, Ertem AG, Yayla KG, Algül E, Yayla Ç, Ünal S, Bilgin M, Çimen T, Kirbaş Ö, Yeter E. Serum Irisin Level Can Predict the Severity of Coronary Artery Disease in Patients with Stable Angina. Korean Circ J 2017; 47 (1): 44-49.

11. Baran A, Myśliwiec H, Kiluk P, Świderska M, Flisiak I. Serum irisin levels in patients with psoriasis. J Dermatolog Treat 2017; 28 (4): 304-308.

12. Icli A, Cure E, Cumhur Cure M, Uslu AU, Balta S, Arslan S, Sakiz D, Kucuk A. Novel myokine: irisin may be an independent predictor for subclinic atherosclerosis in Behçet's disease. J Investig Med 2016; 64 (4): $875-881$.

13. Stengel A, Hofmann T, Goebel-Stengel $M$ et al. Circulating levels of irisin in patients with anorexia nervosa and different stages of obesity - correlation with body mass index. Peptides 2013; 39: 125-130.

14. Moreno-Navarette JM, Ortega F, Serrano M et al. Irisin is expressed and produced by human muscle and adipose tissue in association with obesity and insulin resistance. J Clin Endocrinol Metab 2013; 98: E769-778.

15. Sanchiz-Gomar Lippi G, Mayero $\mathbf{S}$ et al. Irisin: a new potential hormonal target for the treatment of obesity and type 2 diabetes. J Diabet 2012; 4: 196.

16. Liu JJ, Wong MD, Toy WC. Lower circulating irisin is associated with type 2 diabetes mellitus. J Diabet Compl 2013; 27: 365-369.

17. Büyüktuna SA, Doğan HO, Bakir D, Erşan S, Koç D, Bakir S. Increased Irisin Concentrations in Patients with Crimean- Congo Hemorrhagic Fever. Jpn J Infect Dis 2017; 70 (5): 475-478. 